\title{
Optical Gain in GaInNAs and GaInNAsSb Quantum Wells
}

\author{
James W. Ferguson, Peter Blood, Fellow, IEEE, Peter M. Smowton, Member, IEEE, Hopil Bae, \\ Tomas Sarmiento, Student Member, IEEE, James S. Harris, Fellow, IEEE, Nelson Tansu, Senior Member, IEEE, \\ and Luke J. Mawst, Senior Member, IEEE
}

\begin{abstract}
We have measured the absorption, gain and spontaneous emission spectra of GaInNAsSb $(3.3 \% \mathrm{~N})$, GaInNAs $(0.5 \% \mathrm{~N})$ and GaInAs quantum well structures to compare their merits as laser gain media. The parameters describing the relations between peak gain and current provide only limited insight. From the analysis of absorption spectra we have determined the intrinsic properties of the structures, represented by the product [reduced density of states $x$ matrix element $x$ overlap integral], taking account of differences in operating wavelength, well width and confinement. We find only a small variation in this product across the samples. The GaInNAsSb structure has a low radiative recombination current due in part to its low photon energy and also to differences in conduction and valence band densities of states and less inhomogeneous broadening relative to GaInNAs. We speculate that $\mathrm{Sb}$ brings benefits as a surfactant producing more homogeneous wells so $\mathrm{Sb}$ may also be beneficial in structures at shorter wavelength. However, there is a large nonradiative current in GaInNAsSb and achieving further reductions in the non-radiative current is the major challenge in taking advantage of the good gain potential of this system.
\end{abstract}

Index Terms-Dilute nitrides, optical gain, quantum well lasers.

\section{INTRODUCTION}

$\mathbf{T}$ HE many decades of development of semiconductor laser diodes have shown continual reductions in threshold current density and this basic device characteristic remains an important figure of merit of new active region materials and structures. However, taken alone, the threshold current density offers little guidance on the potential for improvements in "material quality" or intelligent design of the overall device structure. More detailed information in the form of the relation

Manuscript received January 12, 2011; revised March 3, 2011; accepted March 6, 2011. Date of current version May 17, 2011. This work was supported in part by the Engineering and Physical Sciences Research Council, U.K., under Grant EP/F006683 and in part by Oclaro Inc., for CASE studentship.

J. W. Ferguson, P. Blood, and P. M. Smowton are with the School of Physics and Astronomy, Cardiff University, Cardiff CF24 3AA, U.K. (e-mail: fergusonjw@cf.ac.uk; bloodp@cf.ac.uk; smowtonpm@cf.ac.uk).

H. Bae, T. Sarmiento, and J. S. Harris are with the Solid State Photonics Laboratory, Stanford University, Stanford, CA 94305 USA (e-mail: hpbae@stanford.edu; tsarmie@stanford.edu; harris@snow.stanford.edu).

N. Tansu is with the Centre for Optical Technologies, Department of Electrical and Computer Engineering, Lehigh University, Bethlehem, PA 18015 USA (e-mail: tansu@lehigh.edu).

L. J. Mawst is with the Department of Electrical and Computer Engineering, Reed Centre for Photonics, University of Wisconsin-Madison, Madison, WI 53706 USA (e-mail: mawst@engr.wisc.edu).

Color versions of one or more of the figures in this paper are available online at http://ieeexplore.ieee.org.

Digital Object Identifier 10.1109/JQE.2011.2129492 between peak gain and current density is a valuable tool for optimisation of cavity length and facet reflectivity and the parameters commonly used to describe this curve are sometimes used as figures of merit when comparing active regions with different material systems. Although the influence of the waveguide can be taken into account, these data do not provide insight into the intrinsic capability of an optical gain medium, particularly for systems operating at different wavelength.

A topic of current interest is the use of dilute GaInNAs alloys to produce quantum well structures on GaAs substrates emitting at wavelengths of $1.3 \mu \mathrm{m}$ and beyond [1]. The addition of $\mathrm{Sb}$ has enabled the emission wavelength of this system to be extended to the $1.5 \mu \mathrm{m}$ region [2], [3]. Further refinements have included the use of GaNAs barriers to compensate the large amount of strain in the wells [4]. With these developments it has been possible to produce devices with threshold current densities of $200 \mathrm{~A} \mathrm{~cm}^{-2}$ at $1.3 \mu \mathrm{m}$ [5] and as low as $373 \mathrm{Acm}^{-2}$ for a $3 \mathrm{~mm}$ long device at $1.55 \mu \mathrm{m}$ [3] respectively. Through the interaction of the $\mathrm{N}$ defect level with the conduction band dispersion curve of the host material the band structure is modified by the addition of nitrogen to GaInAs, bringing about changes in effective mass, matrix element and density of states [6]. However calculations show that the band structure is sensitive to the statistical distribution and local environment of the nitrogen atoms and the formation of N-N pairs and clusters [7]. Furthermore the manner of incorporation of nitrogen is influenced by the use of $\mathrm{Sb}$ as a surfactant, as well as the direct effect of incorporated $\mathrm{Sb}$ on the band structure, though the concentration incorporated into the crystal is not necessarily known. Thus it is hard to predict the band structure and matrix element of specific samples since the $\mathrm{N}$ and $\mathrm{Sb}$ concentrations, both of which are small, may not be known with sufficient accuracy and the $\mathrm{N}$ distribution is not known. Additionally, these elements bring about changes in the emission wavelength which itself influences the optical gain and recombination rates, so the aim of this work is to analyse experimental data to identify changes in gain-current characteristics which arise directly from the intrinsic electronic band structure and indirectly from "external" factors such as differences in well width, waveguide characteristics, and emission wavelength (while recognising that the latter arises from the intrinsic band structure).

This question has been addressed by analysis of measurements of gain, absorption and emission spectra on three 
different quantum well structures comprising GaInAs at $1.2 \mu \mathrm{m}$ (as a reference), GaInNAs at $1.3 \mu \mathrm{m}$ and GaInNAsSb at $1.55 \mu \mathrm{m}$; some data on GaInAs and GaInNAs has been reported previously [8]. This analysis shows that the parameters representing the measured gain-current curves alone do not provide a reliable means of comparing the gain-producing potential of these structures.

\section{EXPERIMENTAL Details}

The GaInNAsSb wafer was grown by molecular beam epitaxy (MBE) and comprised a single 7nm GaInNAsSb (41\% In, $3.3 \% \mathrm{~N}, 3 \% \mathrm{Sb}$ ) well with $20 \mathrm{~nm}$ GaNAs barriers either side; the waveguide core is $440 \mathrm{~nm}$ thick and $\mathrm{Al}_{0.33} \mathrm{Ga}_{0.67} \mathrm{As}$ provides the waveguide cladding [3]. After growth the structure was annealed at $680{ }^{\circ} \mathrm{C}$ for $10 \mathrm{mins}$ in a rapid thermal annealer using a GaAs proximity cap. The high performance GaInNAs [9] and GaInAs [10] samples were grown using metal organic vapour phase epitaxy (MOVPE) and consist of a $6 \mathrm{~nm} \mathrm{GaIn}(\mathrm{N})$ As $(0$ or $0.5 \% \mathrm{~N})$ well with $7.5 \mathrm{~nm}$ GaAsP barriers within a $300 \mathrm{~nm}$ wide waveguide core with AlGaAs cladding layers. The use of strain-compensated GaAsP leads to strain-compensated GaInNAs and GaInAs quantum well active regions. The compressive strain in all the samples studied is similar $(2-2.5 \%)$ although, as the compositions are not known exactly, we cannot rule out small differences with consequent effects on the band structure. The optical confinement factors and mode index were determined using a standard slab waveguide computation using the nominal dimensions and compositions of the structures.

The gain characteristics were measured by the segmented contact technique [11] using pulsed excitation of 50 micron wide oxide-isolated stripe geometry structures at $300 \mathrm{~K}$. This single-pass measurement produces modal gain, absorption and spontaneous emission rate spectra. The spontaneous emission spectra were calibrated [11] and integrated to obtain the radiative current density. There was no measureable TM emission from these samples (none is expected due to the strain) and all measurements were made for TE polarised light. The modal absorption spectrum and the gain spectra give the internal optical mode loss $\left(\alpha_{i}\right)$, the photon energy of the half height of the absorption edge $\left(E_{a b s}\right)$ and the transparency photon energy $\left(E_{t}\right)$ at each current.

\section{Gain-CurRent Relation}

Fig. 1 shows measured values of peak modal gain obtained from the gain spectra (the measured net modal gain plus $\alpha_{i}$ ) as functions of the measured applied current density $\left(J_{t o t}\right)$ (upper plot) and the radiative current density $\left(J_{r a d}\right)$ calculated from the measured spontaneous emission spectra for contributions from two TE transitions (lower plot). (Data in the form of Fig. 1 is sometimes obtained from measurements of threshold current density for different cavity lengths.) Additional gain versus radiative current density measurements for the GaIn$\mathrm{NAsSb}$ material were taken on a device with three quantum wells to provide results at a lower injection per well. Our previous work [12] showed that the wells in these three and one well samples were similar and that both the peak gain
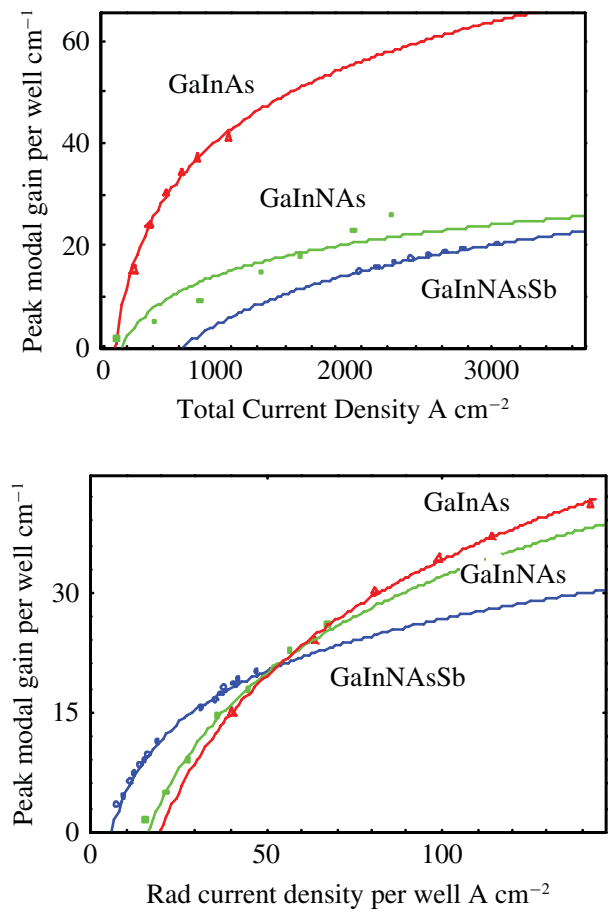

Fig. 1. Measured values of peak modal gain as a function of total drive current density for single well structures (upper) and peak modal gain per well as a function of radiative current density per well (lower). The lines are fits of (1) with parameters given in Table I.

and radiative current density scale with well number so the results in the lower plot of Fig. 1 are given as modal gain per well and radiative current density per well. The total current density does not scale with well number, due to current flow by recombination in the barriers, so the three well data is not included in the upper plot of Fig. 1, where all the results refer to a single well. The gain from a single pair of sub-bands can be represented by the analytic approximation due to McKilroy et al [13] in the equivalent modified form [14]

$$
G_{p k}=G_{0} \ell n\left(\frac{J}{J_{\text {trans }}}\right) .
$$

Here $G_{p k}$ represents the peak modal gain for the spectrum measured at the current density $J ; J_{\text {trans }}$ is the transparency current density (the current density at which gain is first produced where $G_{p k}$ is zero) and $G_{0}$ is the gain parameter which, with $J_{\text {trans }}$, defines the parameterised gain-current characteristic. The values of parameters from these fits to the radiative current density are given in Table I $\left(G_{0}\right.$ and $\left.J_{\text {trans }}^{\text {rad }}\right)$, together with values for the total transparency current density $\left(J_{\text {trans }}^{\text {tot }}\right)$. Comparing the total and radiative transparency currents it is clear that there are non-radiative contributions to the total current in all the samples, particularly those containing nitrogen. Where this non-radiative contribution is due to Shockley-Read-Hall (SRH) recombination it can in principle be eliminated, in which case the radiative current density indicates the intrinsic current density of the structure. In the case of GaInNAsSb wells our previous work [12] suggests that the intrinsic Auger recombination current density in the well may be significantly larger than the radiative 
TABLE I

Gain-Current Curve Parameters Obtained by Fitting (1) to the Modal and Local Gain ( $G_{0}$ AND $g_{0}$ Respectively) IN FIGURES 1 AND 2

\begin{tabular}{|c|c|c|c|c|}
\hline $\begin{array}{c}\text { Sample } \\
\text { nitrogen } \\
\text { wavelength }\end{array}$ & $\begin{array}{c}\text { J }_{\text {trans }}^{\text {radians }} \\
\mathrm{A} \mathrm{cm}^{-2}\end{array}$ & $\begin{array}{c}\text { J }_{\text {trans }}^{\text {tot }} \\
\mathrm{A} \mathrm{cm}^{-2}\end{array}$ & $\begin{array}{c}G_{0} \\
\text { Modal } \\
\text { gain } \\
\mathrm{cm}^{-1}\end{array}$ & $\begin{array}{c}g_{0} \\
\text { local } \\
\text { gain } \\
\mathrm{cm}^{-1}\end{array}$ \\
\hline $\begin{array}{c}\text { GaInAs, } \\
1.2 \mu \mathrm{m}\end{array}$ & 19.8 & 104 & 21.1 & 1240 \\
\hline $\begin{array}{c}\text { GaInNAs } \\
0.5 \% \mathrm{~N} \\
1.3 \mu \mathrm{m}\end{array}$ & 16.1 & 156 & 17.5 & 1014 \\
\hline $\begin{array}{c}\text { GaInNAsSb } \\
3.3 \% \mathrm{~N}, \\
1.55 \mu \mathrm{m}\end{array}$ & 5.94 & 622 & 9.5 & 993 \\
\hline
\end{tabular}

current density, and simulations also show a substantial nonradiative current due to carriers thermally excited to the adjacent barrier material. We have estimated that if SRH recombination could be eliminated a threshold current density of $320 \mathrm{~A} \mathrm{~cm}^{-2}$ (Radiative + Auger) could be achieved in a similar structure for a $1 \mathrm{~mm}$ long device [12]. The experimental gain data of Thranhardt et al [15] also shows that a higher drive current is required to produce similar peak gain from $\mathrm{GaInN}_{0.0043} \mathrm{As}_{0.9957}$ and GaInAs wells (by a factor of about 2).

While the modal gain determines the threshold current, the local gain, if correctly used, may be more useful as a comparator since it is an intrinsic property of the gain medium. The modal gain for a single sub-band is only weakly dependent on well width and the expression for local gain, $g$, contains an artificial well width dependence which is compensated by the well width dependence of the confinement factor [16]. To remove this dependence we have calculated the local gain from the measured modal gain using the confinement factor for each waveguide structure each with a well width of $6 \mathrm{~nm}$. This is given in Fig. 2 as a function of the radiative current density. The values of the local gain parameter, $g_{0}$, obtained by fitting the same form as equation (1) are given in Table I and if this parameter is used as an intrinsic figure of merit it suggests a superior gain potential of the GaInAs structure with $g_{0}=1240 \mathrm{~cm}^{-1}$ followed by GaInAsN and GaInNAsSb in order of descending values of $g_{0}$.

A shortcoming of equation (1) is that the peak gain does not approach an asymptotic value at high current whereas the gain from a single pair of sub-bands tends to a saturation value at high current due to the form of the joint density of states function. While the saturation value of the gain, $g_{\text {sat }}$ is not usually accessible experimentally due to the onset of gain from the next pair of sub-bands it does provide a measure of the maximum gain which a single pair of sub-bands can provide. In principle $g_{s a t}$ can be determined by fitting an alternative expression to the $g-J$ data, originally proposed for quantum dot systems [17]

$$
g_{p k}=g_{s a t}\left\{1-\exp \left[\frac{-\gamma\left(J-J_{\text {trans }}\right)}{J_{\text {trans }}}\right]\right\}
$$

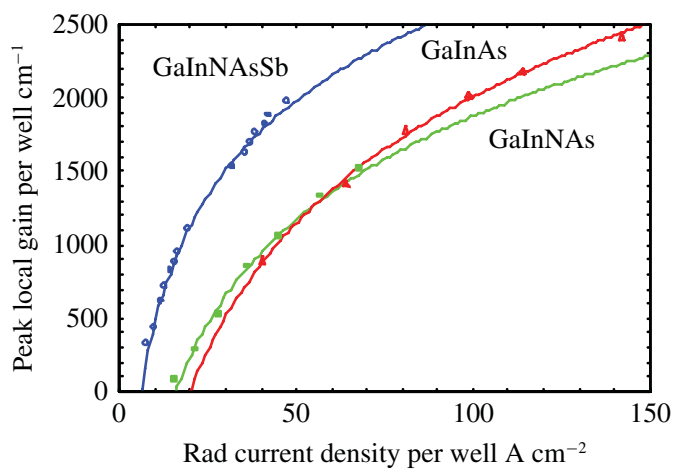

Fig. 2. Local peak gain for a $6 \mathrm{~nm}$ wide well as a function of radiative current density per well, obtained from the modal gain data in Fig. 1. The lines are fits of (1) with the parameters given in Table I.

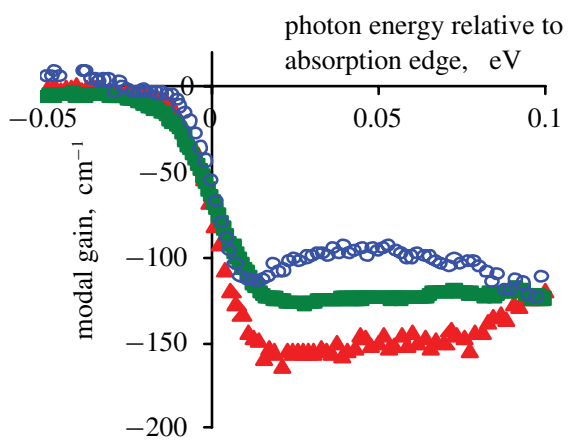

Fig. 3. Measured modal absorption spectra as a function of photon energy plotted relative to the band edge energy for each structure. Triangles: GaInAs, squares: GaInNAs, open circles: GaInNAsSb.

where $\gamma$ is a further adjustable parameter which ideally is close to unity. We have tested equation (2) for wells by fitting it to a model $g$ - $J$ relation calculated for a single pair of sub-bands of a GaInAs well and find that it is not a good representation of the gain current relation and the values of $g_{\text {sat }}$ obtained depend upon the data range over which the fit is performed.

\section{Optical Absorption Spectra}

A fundamental measure of the strength of the interaction with light is provided by the optical absorption spectra, which are shown in Fig. 3. The values of internal optical mode loss, (determined by averaging values taken from both gain and absorption spectra in the region where the net absorption and gain is independent of photon energy) have been subtracted from the magnitude of the net absorption to produce these data and the spectra have been plotted relative to the photon energy at their respective band edges $\left(E_{a b s}\right)$. If we assume that many-body interactions produce similar enhancement effects under conditions for laser operation in all the samples then the relative values of modal absorption indicate the relative maximum gains which can be generated in these waveguides under full inversion. Figure 3 suggests that GaInAs has the superior modal gain capability, which is consistent with the values of $G_{0}$, though the value of $G_{0}$ for GaInNAsSb (Table I) is lower relative to GaInAs than suggested by the modal absorption near the band edge in Fig. 3 . 
TABLE II

Values of the Local absorption Coefficient in the Plateau Region, 10-20 meV Above the Band Edge, Obtained From the Results in Fig. 3, and Values of the Parameter $Y$ Derived From It (4). Values Are Also Given for the Population Inversion Factor $P_{f}$ (5) at the Gain Peak, the Photon Energy of the Gain Peak, and the Transparency Energy (the Latter Two Parameters Being Given Relative to the Absorption Edge) All for a Peak Local Gain of $860 \mathrm{CM}^{-1}$

\begin{tabular}{|c|c|c|c|c|c|}
\hline $\begin{array}{c}\text { Sample } \\
\text { nitrogen } \\
\text { wavelength }\end{array}$ & $\begin{array}{c}n \\
\text { effective } \\
\text { index }\end{array}$ & $\begin{array}{c}\alpha \text { local } \\
\text { absorption } \\
\mathrm{cm}^{-1}\end{array}$ & $Y \mathrm{~m}^{-2} \mathrm{~kg}$ & $P_{f}$ & $\begin{array}{c}\text { Transparency energy } \\
\text { relative to } \\
E_{a b s}( \pm 0.5) \mathrm{meV}\end{array}$ \\
\hline GaInAs, $1.2 \mu \mathrm{m}$ & 3.23 & 8940 & $7.410^{-13}$ & 0.54 & 29 \\
\hline $\begin{array}{c}\text { GaInNAs } 0.5 \% \mathrm{~N} \\
1.3 \mu \mathrm{m}\end{array}$ & 3.23 & 7290 & $5.710^{-13}$ & 0.59 & 35 \\
\hline $\begin{array}{c}\text { GaInNAsSb } \\
3.3 \% \mathrm{~N}, 1.55 \mu \mathrm{m}\end{array}$ & 3.28 & 11000 & $7.310^{-13}$ & 0.83 & 24 \\
\hline
\end{tabular}

To isolate the influence of the intrinsic properties of the structures, we have calculated the local material absorption coefficient, $\alpha$, from the modal absorption in the plateau region 0.01 to $0.02 \mathrm{eV}$ above the band edge for a well width of $6 \mathrm{~nm}$ (Table II) and find that the local absorption of the GaInNAsSb structure is higher than that of the others. The absorption coefficient for a single pair of sub-bands is given by [16]

$$
\begin{aligned}
\alpha= & \frac{4 \pi \hbar}{n c \varepsilon_{0}(\hbar \varpi)}\left(\frac{e}{2 m_{0}}\right)^{2} \\
& \times\left\{\left|M^{p}\right|^{2} \times\left\{\int F_{0}^{*}(z) F_{c}(z) d z\right\}^{2} \rho_{\text {red }}\right\} \frac{1}{L_{z}}
\end{aligned}
$$

where $F_{c}$ and $F_{v}$ are the envelope functions of the confined states in the conduction and valence sub-bands, $\rho_{\text {red }}$ is the reduced density of states per unit area, and $M^{p}$ is the momentum matrix element for transitions of polarisation $p$. This equation associates emission at a particular photon energy with transitions between a well-defined pair of states and therefore assumes that the joint density of states is slowly varying so that the effect of homogeneous broadening is small. (This is not true immediately at the band edge.) Using the values of $\alpha$ in Table II and the corresponding photon energy we have calculated values for the parameter

$$
Y=\left\{\left|M^{p}\right|^{2}\left\{\int F_{v}^{*}(z) F_{c}(z) d z\right\}^{2} \rho_{\text {red }}\right\}
$$

which is a measure of the overall strength of the interaction of the structure with light, independent of the photon energy. The values obtained for this parameter (in $\left.\mathrm{kg} \mathrm{m}^{-2}\right)( \pm 8 \%)$ are GaInAs $7.4 \times 10^{-13}$, GaInNAs $5.7 \times 10^{-13}$ (lowest) and GaInNAsSb $7.3 \times 10^{-13}$.

From these data we cannot determine the origin of differences in the values of $Y$ determined from the absorption spectra. From k.p theory, when the effective mass $\left(m^{*}\right)$ is small compared with the free electron mass we expect the product $\left(m^{*} \times M^{2}\right)$ to remain approximately constant ([14] eqn A8.14) as $\mathrm{N}$ is added so that $\rho_{\text {red }}|M|^{2}$ should not change significantly between GaInAs and GaInAsN. Tomic and O'Reily comment that although addition of $2 \%$ nitrogen is calculated to reduce $\left|M_{T}\right|^{2}$ by about $30 \%$ the calculated joint optical density of states (and hence the absorption coefficient) shows a weak variation [6]. We estimate a reduction of about $23 \%$ in $Y$ with the addition of a nominal $0.5 \% \mathrm{~N}$ to GaInAs, while the values for GaInNAsSb and GaInAs are similar. The overlap integral may be different between the structures and it is not clear whether it is included in the calculations of $\left|M_{T}\right|^{2}$ in ref [6] though as defined by equation 4.21 of ref [14] this would be the case.

The absorption spectrum of the Sb-containing sample (Fig. 3) suggests that its density of states function may be peaked near the band edge in contrast to the other two structures. The GaInNAs structure has greater broadening of the absorption edge (Fig. 3) which may be due to inhomogeneous broadening due to well width or composition variations. It has been reported that a higher inhomogeneous broadening is required to fit gain spectra of GaInNAs quantum wells than GaInAs wells (17 meV compared to $11 \mathrm{meV}$ ) [15]. The role of $\mathrm{Sb}$ as a surfactant is to inhibit phase segregation and roughening which should reduce inhomogeneous broadening in the GaInNAsSb structure.

From values of local absorption coefficient in Table II (for the same well width) we conclude that the GaInNAsSb structure has highest gain-producing potential, even though it has a similar value of the intrinsic parameter $Y$ to GaInAs; this arises largely as a result of its low photon energy (equation 3 ).

\section{SpontaneOUS EMISSION}

A high gain capability must be assessed against the current required to achieve it, and the radiative recombination rate is also determined by the intrinsic factors within the quantity $Y$. However, we note that the GaInNAsSb sample has a similar value of $Y$ to GaInAs but the lowest radiative transparency current density of all three samples (0.36 that of GaInAs, Table I). However the structures have significant differences in photon energy and index which influence the relation between gain and recombination rate. Furthermore the total radiative current is obtained by integrating the spontaneous emission spectrum over the thermal carrier distribution and over all directions and polarizations, though in all these samples the TM emission is negligible. While there are substantial nonradiative contributions to the current, the emission spectra give insight into the width of the carrier distribution which is relevant to other recombination processes. The carrier distribution 

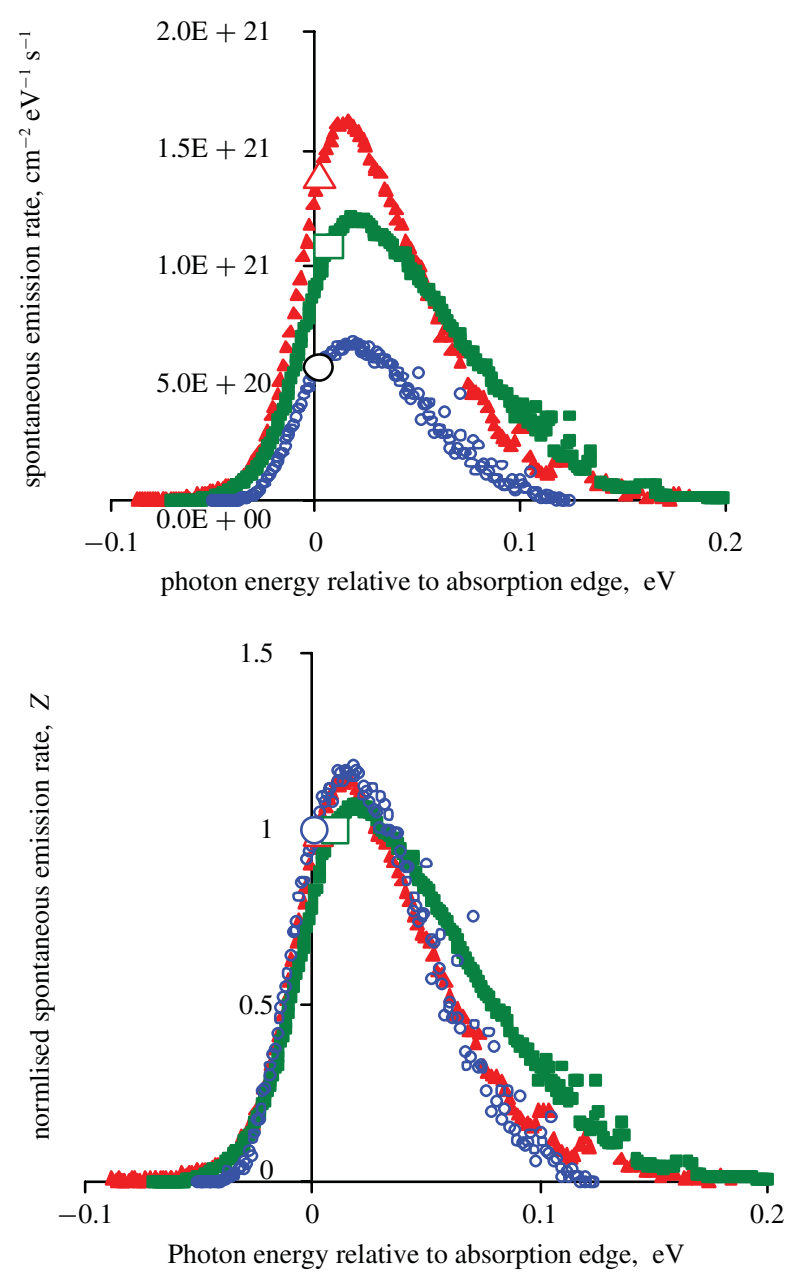

Fig. 4. Upper plot: Spontaneous emission spectra at a current for a local material gain of $860 \mathrm{~cm}^{-1}$ in each structure relative to the absorption edge. The large open symbols denote the data point at the peak gain; triangles: GaInAs, squares: GaInNAs, open circles: GaInNAsSb. Lower plot: Normalised spontaneous emission spectra: the parameter $Z,(6)$.

at a given temperature is determined by the form of the density of states functions for electrons and holes and the quasi Fermi level positions relative to the band edges determined by the requirement of charge neutrality. All these factors influence the spectrally integrated radiative recombination rate, in addition to factors in the parameter $Y$.

The spontaneous emission spectra for emission into a single TE mode measured at the drive current which produces a peak gain of about $860 \mathrm{~cm}^{-1}$ (for a $6 \mathrm{~nm}$ well) in each sample are shown in Fig. 4 plotted relative to the absorption edge of each sample. The large open symbols show the data points corresponding to the gain peak in each case. Under carrier injection many-body effects shift the gain and emission spectra relative to the absorption spectra, however our data suggests these shifts are similar in all three structures so we can effect a comparison between their spectra by plotting them relative to the absorption edge energy $\left(E_{a b s}\right)$. The spectra in the upper plot of Fig. 4 show clearly that the differences in total radiative current arise from differences in the magnitude of the radiative rate at the gain peak (for the same peak gain) and from differences in the spectral distributions.

\section{A. Spontaneous Emission at the Gain Peak}

The local gain and spontaneous emission rate at a given photon energy are related by [11]

$$
r_{\text {spon }}(\hbar \varpi)=\frac{n^{2}(\hbar \varpi)^{2}}{3 \pi^{2} \hbar^{3} c^{2}}\left[\frac{f_{1}\left(1-f_{2}\right)}{\left(f_{1}-f_{2}\right)}\right] g(\hbar \varpi)
$$

which is independent of the parameter $Y$. (The matrix element cancels exactly in this case because all the emission is in the same polarization as the gain so there is no average over polarization in the calculation of the total emission rate.) The occupation factors of the upper and lower states participating in the transition are $f_{1}$ and $f_{2}$. The emission rates at the gain peak, for the same peak gain, (Fig. 4, large open symbols) are different for each structure and their ratios relative to GaInAs (0.74 and 0.40 for GaInNAs and GaInNAsSb respectively) are not simply accounted for by the factor $\left\{n^{2}(h \varpi)^{2}\right\}(0.91$ and 0.63 relative to GaInAs respectively) which implies there are also differences in the statistical factor in square brackets. This term is the reciprocal of population inversion factor $P_{f}$ [11], and values of $P_{f}$ at the gain peak energy computed from the measured values of gain and emission rate using equation (5) are given in Table II. We conclude that the ratios of the spontaneous emission rates at the gain peak for the same peak gain, are due to a combination of factors: mode index, photon energy and population inversion factor, all of which culminate in a significant reduction in radiative current for GaInNAsSb.

It is instructive to explore the reasons for the variations in the statistical factor for the same peak gain between the samples. The inversion factor $P_{f}$ is determined by the individual occupation factors of the pair of states participating in the transition, which in turn are determined by charge neutrality which specifies the individual electron and hole quasi Fermi level energies. The densities of states of the conduction and valence bands must be such as to bring about relative values of $f_{1}$ and $f_{2}$ which produce the high inversion factor in GaInNAsSb. We can estimate the quasi Fermi level separation necessary to achieve a gain of $860 \mathrm{~cm}^{-1}$ from the photon energy at which the gain is zero (the transparency energy $E_{t}$ ). The quasi-Fermi level separation relative to the band gap is an indication of the inversion of each system and if manybody shifts are similar for all three structures we can use the absorption edge $\left(E_{a b s}\right)$ as a reference to remove differences in the band gap. The values of the transparency energies for a peak gain of $860 \mathrm{~cm}^{-1}$, relative to the respective absorption edge, are given in Table II and indicate that the GaInNAsSb structure achieves this gain with a marginally lower relative quasi-Fermi level separation than the other structures. This may be a consequence of its sharper band edge as indicated by the absorption spectra in Fig. 3.

\section{B. Spontaneous Emission Spectra}

Finally, we make a comparison of the spontaneous emission spectra at the drive current necessary for a gain of $860 \mathrm{~cm}^{-1}$. We do this by plotting the spontaneous emission spectrum of each structure relative to its spontaneous emission rate at the gain peak energy $\left(h \varpi_{p k}\right)$ and since emission occurs over 
different spectral regions for each structure we take account of differences in photon energy by calculating the ratio:

$$
Z\left(\hbar \varpi-E_{a b s}\right)=\frac{R_{\text {spon }}(\hbar \varpi)}{R_{\text {spon }}\left(\hbar \varpi_{p k}\right)} \frac{\hbar \varpi_{p k}}{\hbar \varpi}
$$

as plotted in Fig. 4. This shows how the total radiative currents are determined by the relative shapes of the spectra irrespective of the wavelength region. The ratios of the areas of the normalised spectra in Fig. 4 relative to GaInAs $=1$ are GaInNAs 1.12 and GaInNAsSb 0.95 . The consequences of the low relative quasi Fermi level separation for GaInNAsSb are apparent, in contrast to the broader spectrum for GaInNAs which may also be related to inhomogeneous broadening of the band edge in this sample, noted in section IV.

\section{DISCUSSION}

From the values of the local gain parameter $g_{0}$ it could be concluded that the GaInAs structure has a marginally superior gain capability compared with the other structures, in contrast to the local absorption coefficient which is greatest for GaInNAsSb. The gain parameter is determined as the tangent to the gain-current curve through the origin [13] and, while $G_{0}$ is independent of multiplicative scaling of the current, in general its value is influenced by the transparency current: the lower the transparency current the lower the values of $G_{0}$ and $g_{0}$ for the same maximum gain at high current (in the limit as $J_{\text {trans }} \rightarrow 0, g_{0} \rightarrow 0$ ). While these parameters provide an analytic means of optimising a device structure, the gain parameter is not uniquely related to the gain capability of the system.

The absorption coefficient is a direct indicator of the light-matter interaction and the occupation factors are known $\left(f_{1}=0, f_{2}=1\right)$ provided the measurement is made at low excitation. The material absorption coefficient $\alpha$ is greatest for GaInNAsSb indicating that this system has the potential to provide the largest maximum gain. Values of the [matrix element $\times$ reduced density of states $\times$ overlap integral] product $(Y)$ from our measurements yield a value for GaInNAsSb which is similar to GaInAs, with the lower value for GaInNAs only just outside the experimental uncertainty. Thus the observed differences in absorption coefficient arise principally from differences in "external" factors: photon energy and mode index, rather the intrinsic band structure, measured by $Y$.

We expect the reduced density of states to increase with $\mathrm{N}$ content going down the list in Table II due to the increase in mass, however the momentum matrix element generally decreases with decreasing band gap and it is predicted that the local absorption coefficient is only weakly affected by addition of $N$ [6]. Our results are broadly consistent with this within the measurement uncertainty.

Despite similar values for the intrinsic parameter $Y$, the low recombination current in the GaInNAsSb structure is in part due to its low photon energy and high population inversion factor; the latter may also be intrinsic, arising from differences in the relative conduction and valence band densities of states. A contribution to the wider emission spectrum of GaInNAs may be greater inhomogeneous broadening at the band edge, apparent in the absorption spectra. The sharper band edge in
GaInNAsSb, despite its higher $\mathrm{N}$ content, may be due to $\mathrm{Sb}$ acting as a surfactant influencing the $\mathrm{N}$ incorporation and the lateral homogeneity of the well. It is the combination of these intrinsic and external factors which leads to the low radiative current for GaInNAsSb, while maintaining a higher local gain capability.

The total current in the GaInNAsSb structure is much greater than the radiative current at transparency and previous work suggests that Auger recombination may make a contribution to the non-radiative current. The emission spectra provide an indication of the carrier population and distribution and these also influence non-radiative processes. The spectra in Fig. 4 suggest both a lower carrier density and a narrower distribution in GaInNAsSb than in the other structures, and this must be a factor in moderating the Auger current and any currents related to carrier leakage.

\section{CONCLUSION}

We conclude that the gain-current parameters do not provide reliable insight into the gain-potential of the structures studied. GaInNAsSb has the capability to produce more local gain than other structures at their respective wavelengths due primarily to differences in wavelength and index. The intrinsic [matrix element $\times$ density of states $\times$ overlap integral] is similar for the three stuctures, as predicted. Differences in the densities of states in the conduction and valence bands may contribute to the low radiative current in GaInNAsSb, through the high inversion factor, and the use of $\mathrm{Sb}$ may produce a sharper band edge in GaInNAsSb quantum wells compared with GaInNAs, benefitting the spectrally integrated recombination rate. If this is so, there may be benefits using $\mathrm{Sb}$ in the growth of structures at shorter wavelength. There is a large non-radiative current in GaInNAsSb and Auger recombination may well be significant though it should be moderated by the relatively low carrier density and narrow carrier distribution. Nevertheless achieving further reductions in the non-radiative current is the major challenge in taking advantage of the good gain potential of this system.

\section{REFERENCES}

[1] N. Tansu, N. J. Kirsch, and L. J. Mawst, "Low-threshold-current-density 1300-nm dilute-nitride quantum well lasers," Appl. Phys. Lett., vol. 81, no. 14, pp. 2523-2525, Sep. 2002.

[2] X. Yang, M. J. Jurkovic, J. B. Heroux, and W. I. Wang, "Molecular beam epitaxial growth of InGaAsN:Sb/GaAs quantum wells for longwavelength semiconductor lasers," Appl. Phys. Lett., vol. 75, no. 2, pp. 178-180, Jul. 1999.

[3] S. R. Bank, H. Bae, L. L. Goddard, H. B. Yuen, M. A. Wistey, R. Kudrawiec, and J. S. Harris, Jr., "Recent progress on $1.55-\mu \mathrm{m}$ dilutenitride lasers," IEEE J. Quantum Electron., vol. 43, no. 9, pp. 773-785, Sep. 2007.

[4] N. Tansu, J.-Y. Yeh, and L. J. Mawst, "Low-threshold 1317-nm InGaAsN quantum-well lasers with GaAsN barriers," Appl. Phys. Lett., vol. 83, no. 13, pp. 2512-2514, Sep. 2003.

[5] N. Tansu and L. J. Mawst, "Current injection efficiency of InGaAsN quantum-well lasers," J. Appl. Phys., vol. 97, no. 5, pp. 054502-1054502-18, Mar. 2005.

[6] S. Tomić and E. P. O'Reilly, "Gain characteristics of ideal dilute nitride quantum well lasers," Phys. E: Low-Dimens. Syst. Nanostruct., vol. 13, nos. 2-4, pp. 1102-1105, Mar. 2002.

[7] E. P. O'Reilly, A. Lindsay, P. J. Klar, A. Polimeni, and M. Capizzi, "Trends in the electronic structure of dilute nitride alloys," Semicond. Sci. Technol., vol. 24, no. 3, pp. 033001-1-033001-11, 2009. 
[8] D. J. Palmer, P. M. Smowton, P. Blood, J. Y. Yeh, L. J. Mawst, and N. Tansu, "Effect of nitrogen on gain and efficiency in InGaAsN quantumwell lasers," Appl. Phys. Lett., vol. 86, no. 7, pp. 071121-1-071121-3, Feb. 2005.

[9] N. Tansu, A. Quandt, M. Kanskar, W. Mulhearn, and L. J. Mawst, "Highperformance and high-temperature continuous-wave-operation $1300 \mathrm{~nm}$ InGaAsN quantum well lasers by organometallic vapor phase epitaxy," Appl. Phys. Lett., vol. 83, no. 1, pp. 18-20, Jul. 2003.

[10] N. Tansu, J. Y. Yeh, and L. J. Mawst, "High-performance 1200-nm InGaAs and 1300-nm InGaAsN quantum-well lasers by metalorganic chemical vapor deposition," IEEE J. Sel. Topics Quantum Electron., vol. 9, no. 5, pp. 1220-1227, Sep.-Oct. 2003.

[11] P. Blood, G. M. Lewis, P. M. Smowton, H. Summers, J. Thomson, and J. Lutti, "Characterization of semiconductor laser gain media by the segmented contact method," IEEE J. Sel. Topics Quantum Electron., vol. 9, no. 5, pp. 1275-1282, Sep.-Oct. 2003.

[12] J. W. Ferguson, P. M. Smowton, P. Blood, H. Bae, T. Sarmiento, and J. S. Harris, Jr., "Nonradiative recombination in $1.56 \mu \mathrm{m}$ GaInNAsSb/GaNAs quantum-well lasers," Appl. Phys. Lett., vol. 95, no. 23, pp. 231104-1231104-3, Dec. 2009.

[13] P. M. McIlroy, A. Kurobe, and Y. Ueematsu, "Analysis and application of theoretical gain curves to the design of multi-quantum-well lasers," IEEE J. Quantum Electron., vol. 21, no. 12, pp. 1958-1963, Dec. 1985.

[14] L. A. Coldren and S. W. Corzine, Diode Lasers and Photonics Integrated Circuits (Microwave and Optical Engineering), K. Chang, Ed. New York: Wiley, 1985.

[15] A. Thranhardt, I. Kuznetsova, C. Schlichenmaier, S. W. Koch, L. Shterengas, G. Belenky, J.-Y. Yeh, L. Mawst, N. Tansu, J. Harder, J. V. Maloney, and W. W. Chow, "Nitrogen incorporation effects on gain properties of GaInNAs lasers: Experiment and theory," Appl. Phys. Lett., vol. 86, no. 20, pp. 201117-1-201117-3, May 2005.

[16] P. Blood, "On the dimensionality of optical absorption, gain, and recombination in quantum-confined structures," IEEE J. Quantum Electron., vol. 36, no. 3, pp. 354-362, Mar. 2000.

[17] A. E. Zhukov, A. R. Kovsh, V. M. Ustinov, A. Y. Egorov, N. N Ledentsov, A. F. Tsatsul'nikov, M. V. Maximov, Y. M. Shernyakov, V. I. Kopchatov, A. V. Lunev, P. S. Kop'ev, D. Bimberg, and Z. I. Alferov, "Gain characteristics of quantum dot injection lasers," Semicond. Sci. Technol., vol. 14, no. 1, pp. 118-123, 1999.

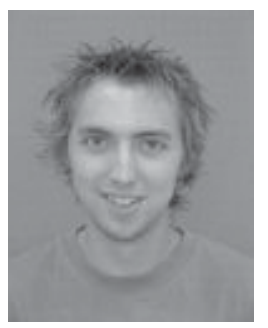

James W. Ferguson received the B.Sc. degree in physics and the Ph.D. degree in optoelectronics from Cardiff University, Cardiff, U.K., in July 2006 and November 2010, respectively.

$\mathrm{He}$ is now working in the defense sector. His current research interests include gain characteristics of dilute nitride quantum well structures.

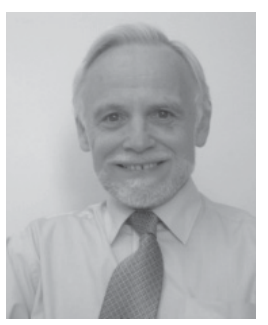

Peter Blood (M'01-F'08) received the Ph.D. degree from the University of Leeds, Leeds, U.K.

He was with the Philips Research Laboratory, Redhill, U.K., working on electrical properties of semiconductors and spent some time as a Visitor with Bell Laboratories, Murray Hill, NJ. He has been investigating quantum well lasers since 1983, and he moved to Cardiff University, Cardiff, U.K., in 1990, where the group has developed observations of spontaneous emission from laser diodes as a means of probing their steady-state and dynamic behavior. His current research interests include gain and recombination in selfassembled quantum-dots and carrier localization in dots. He has given short courses and review tutorials on quantum-confined lasers at major international conferences.

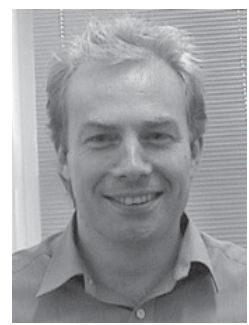

Peter M. Smowton (M'06) received the Ph.D. degree from the University of Wales, Cardiff, U.K., in 1991, where he studied the frequency stabilization of laser diodes.

He was responsible for device processing at the Cardiff Microelectronics Center, Cardiff, from 1991 to 1992. He has been employed in the School of Physics and Astronomy, Cardiff University, Cardiff, since 1993, where he is currently a Professor of Semiconductor Optoelectronics. His current research topics include quantum-dot lasers, recombination mechanisms in dilute nitride devices, high-power red emitters for photodynamic therapy, and the physics of InGaN light-emitting devices. His current research interests include design, fabrication, and characterization of optoelectronic devices.

Hopil Bae received the B.S. degree in electrical engineering from the Seoul National University, Seoul, Korea, and the M.S. and Ph.D. degrees in electrical engineering from Stanford University, Stanford, CA. For his Ph.D., he worked on long wavelength GaInNAsSb-based edge-emitters and vertical cavity surface emitters lasers.

He also worked in the industry as a digital logic designer for wireless communication receiver chips, before beginning his study at Stanford University. Currently, he is working at Apple Inc., Cupertino, CA, as a Display Engineer.

Tomas Sarmiento (S'01) received the B.S. degree in electrical engineering from the University of Los Andes, Bogota, Colombia, in 1998, and the M.S. degree in electrical engineering from the Georgia Institute of Technology, Atlanta, in 2003. He is currently pursuing the Ph.D. degree in electrical engineering at Stanford University, Stanford, CA.

His current research interests include long-wavelength dilute nitride edgeemitting and vertical cavity lasers.

Mr. Sarmiento was awarded the Gerald L. Pearson Memorial Fellowship from Stanford University, and a Fulbright Fellowship.

James S. Harris (S'66-M'68-SM'77-F'88) received the B.S., M.S., and $\mathrm{Ph} . \mathrm{D}$. degrees all in electrical engineering from Stanford University, Stanford, CA, in 1964, 1965, and 1969, respectively.

He joined Rockwell International Science Center, Thousand Oaks, CA, in 1969 , where he was engaged in developing pre-eminent position in GaAs device technology. In 1982, he joined the Solid State Electronics Laboratory, Stanford University, as a Professor of electrical engineering, where he was also the Director of the Solid State Electronics Laboratory from 1984 to 1998, the Director of the Joint Services Electronics Program from 1985 to 1999, and is currently the James and Ellenor Chesebrough Professor of Engineering. He has more than 750 publications and 18 U.S. patents. His current research interests include physics and application of ultrasmall structures and novel materials to new highspeed and spin-based electronic and optoelectronic devices.

Prof. Harris is a Fellow of the American Physical Society, the Optical Society of America, and the Materials Research Society. He received the IEEE Morris N. Liebmann Memorial Award in 2000, the International Compound Semiconductor Conference Walker Medal in 2000, the IEEE Third Millennium Medal, and the Alexander von Humboldt Senior Research Prize in 1998 for contributions to GaAs technology. 


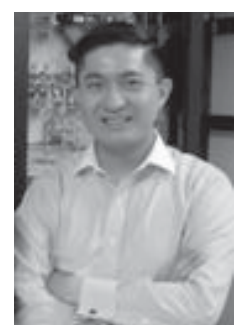

Nelson Tansu (S'99-M'02-SM'10) was born on October 1977. He received the B.S. degree in applied mathematics, electrical engineering, and physics, (with highest distinction) and the Ph.D. degree in electrical engineering from the University of Wisconsin-Madison, Madison, in 1998 and 2003, respectively.

$\mathrm{He}$ is currently an Associate Professor (with tenure) in the Department of Electrical and Computer Engineering, Center for Optical Technologies, Lehigh University, Bethlehem, PA, in 1961. He has published more than 70 refereed journal and 140 conference publications, and holds 8 U.S. patents. His current research interests include low-dimensional semiconductor optoelectronic materials and devices and growth and device fabrication of III-Nitride devices.

Dr. Tansu was the recipient of the Wisconsin Alumni Research Foundation Graduate University Fellowship, the Vilas Graduate University Fellowship, the Graduate Dissertator Award, and the Best Electrical and Computer Engineering Dissertation Award from the University of Wisconsin-Madison. $\mathrm{He}$ is a recipient of the Libsch Early Career Research Award from Lehigh University in 2008, and the Wisconsin Forward Under 40 Award from the University of Wisconsin-Madison in 2010. He was a Primary Guest Editor of the IEEE JOURNAL OF SELECTED TOPICS IN QUANTUM ELECTRONICS from 2008 to 2009, and is an Associate Editor of the IEEE PHOTONICS JOURnAL, Associate Editor for the Optical Society of America Optical Materials Express, and an Associate Editor for Nanoscale Research Letters from 2007 to at present. He was an Invited General Participant at the National Academy of Engineering U.S. Frontiers of Engineering Symposium in 2008, and served on the Organizing Committee for the U.S. Frontiers of Engineering Symposium in 2009.

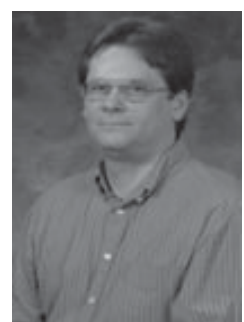

Luke J. Mawst (M'88-SM'93) was born in Chicago, IL, in 1959. He received the B.S. degree in engineering physics and the M.S. and Ph.D. degrees in electrical engineering from the University of Illinois at Urbana-Champaign, Urbana, in 1982, 1984, and 1987, respectively. His dissertation research involved the development of index-guided semiconductor lasers and laser arrays grown by metal organic chemical vapor deposition (MOCVD).

He joined TRW, Inc., Redondo Beach, CA, in 1987, working on the development of semiconductor lasers using MOCVD growth. He is a co-inventor of the resonant optical waveguide antiguided array and contributed to its development as a source of high coherent power. He developed the novel antiresonant-reflective-optical waveguid laser source to couple high power into fibers. He is currently a Professor in the Electrical and Computer Engineering Department, University of Wisconsin-Madison, Madison, involved in the development novel III/V semiconductor device structures, including vertical cavity surface emitters, active photonic structures, dilute-nitride lasers, solar cells, and high-power Al-free diode lasers. He is a founder of Alfalight Inc., Madison, WI, a manufacturer of high power diode lasers. He has authored or co-authored more than 200 technical papers and holds 20 patents.

Prof. Mawst received the TRW Group Level Chairman's Award. 